\title{
Economía turística: Competitividad de servicios hoteleros de Tuxpan, Veracruz
}

Tourism economy: Competitiveness of hotel services in Tuxpan, Veracruz

Elorza Martínez Oralia ${ }^{1}$, Flores Barrios Leonardo ${ }^{1}$, Gómez Priego Ilean A. ${ }^{1}$

Universidad Veracruzana, Facultad de Contaduría. Carretera a la Barra Nte. Kilómetro 7.5, Ejido la

Calzada, C.P. 92880. Tuxpan de Rodríguez Cano, Veracruz.

${ }^{凶}$ Autor para correspondencia: oelorza@uv.mx

Recibido: 04/08/2019

Aceptado: 18/11/2019

\section{RESUMEN}

El puerto de Tuxpan ha tomado auge en los últimos años como destino turístico, el sector hotelero es uno de los principales actores en el escenario turístico por lo que fue el centro de esta investigación. La información fue recabada de medios formales y encuestas realizadas a los prestadores de hoteles que se clasifican con dos y tres estrellas. Los resultados de esta investigación mostraron que los hoteleros encuestados no le otorgan la importancia debida a la competencia, por lo tanto, analizando los resultados obtenidos, se concluye que se hace necesario implementar estrategias para que exista competitividad entre ellos.

Palabras claves: Calidad, Estrategias, Turismo, Servicios, Huéspedes.

\begin{abstract}
The port of Tuxpan has boomed in recent years as a tourist destination, the hotel sector is one of the main players in the tourist scene, which is why it was the center of this research. The information was gathered from formal media and surveys of hotel providers that are classified with two and three stars. The results of this research showed that the hoteliers surveyed do not give due importance to competition, therefore, analyzing the results obtained, it is concluded that it is necessary to implement strategies so that there is competitiveness among them.
\end{abstract}

Keywords: Quality, Strategies, Tourism, Services, Guests. 


\section{INTRODUCCIÓN}

En México el sector hotelero representa el 8.5\% del PIB, uno de los puertos donde el sector hotelero ha tomado auge es el de Tuxpan, Veracruz siendo el tema de la competitividad lo que se abordara desde la perspectiva de la mejora de servicios en general. Los hoteles siempre han puesto en práctica diferentes técnicas para atraer huéspedes como mejorar sus servicios y publicitarse para aumentar sus ingresos, por ello, con el paso del tiempo y el avance de las nuevas tecnologías, los hoteles deben adaptarse a nuevas estrategias de marketing para no perder oportunidades de negocio y ser más competitivos. El sector hotelero se ha sabido amoldar a los tiempos que corren y a las tendencias que va marcando el público, adaptándose a sus gustos y preferencias e intentando captar a más clientes ofreciendo un servicio diferente y mejor.

El sector hotelero pertenece al sector servicios, pero también presenta sus peculiaridades en cuanto a las necesidades de los turistas, los que varían mucho en función de los objetivos principales del viaje, presupuestos, etcétera de ahí que haya tal variedad de servicios hoteleros.

\section{MATERIALES Y MÉTODOS}

Tuxpan de Rodríguez Cano, Veracruz se ubicado entre los paralelos $20^{\circ} 44^{\prime}$ y $21^{\circ} 09^{\prime}$ de latitud norte, y es considerado el tercer puerto de mayor importancia de todo el país. Se realzó una investigación de la industria hotelera regional, específicamente en los hoteles de dos y tres estrellas, se analizó el tipo de servicios y la calidad de los servicios que brindan, la publicidad (mercadotecnia) que utilizan para llegar a los huéspedes. Una vez realizada la investigación se formuló una encuesta que se aplicó a los hoteleros cuyos hoteles se clasifican de dos y tres estrellas. Una vez obtenida la información de los encuestados, los datos fueron analizados para llegar a una conclusión y ofrecer recomendaciones.

El análisis tuvo un alcance descriptivo con enfoque cuantitativo $y$ cualitativo $y$ fue desarrollado de agosto a diciembre del 2018.

\section{RESULTADOS Y DISCUSIÓN}

La investigación fue realizada en el puerto de Tuxpan de Rodríguez Cano, Veracruz, ubicado entre los paralelos $20^{\circ} 44^{\prime}$ y $21^{\circ} 09^{\prime}$ de latitud norte. Colinda al norte con Álamo Temapache, Tamiahua y el Golfo de México; al este con el Golfo de México y Cazones de Herrera; al sur con Cazones de Herrera y Tihuatlán; al oeste con Tihuatlán y Álamo Temapache. En el 2016 fue considerad el tercer puerto de mayor importancia de todo el país. El alcance de esta investigación es descriptivo con enfoque cuantitativo y cualitativo, se desarrolló durante agosto-diciembre 2018 teniendo como objeto de estudio hoteles de dos y tres estrellas del puerto.

Los resultados más relevantes del análisis de los hoteles de dos y tres estrellas sobre la mejora de los servicios para ser competitivos fueron los siguientes:

-Al $50 \%$ de ellos les es indiferente la situación de competitividad hotelera de la región.

-Sólo el $30 \%$ de ellos cree pertinente actualizarse, mejorar sus servicios y ser competentes para atraer más y mejor turismo, brindar a los turistas mejores y variadas opciones 
de alojamiento, y mejorar los ingresos propios y regionales.

-El 65\% de los encuestados mencionaron que los servicios de los hoteles son los necesarios para los huéspedes, sin embargo lo mencionado es negativo.

-El 100\% de los encuestados están conscientes de que sus servicios no son competentes. De acuerdo a la información recabada, se puede mencionar que los hoteleros del área cercana al centro de la ciudad de Tuxpan, Veracruz, carecen de interés y conocimiento para mejorar sus servicios en general (aplicación de mercadotecnia para generar interés en nuevos cliente, mejorar o implementar nuevos servicios, etcétera.).La desmotivación de los prestadores de servicios hoteleros se fundamenta en el hecho de que tienen "ventajas" por ser económicos, que se encuentran relativamente en la zona centro de la ciudad y el resto de los servicios que buscan sus clientes se encuentran en la misma zona. Lo anterior es considerado de mayor relevancia por los clientes, por lo que la ocupación por turistas es segura, pese a que carezcan de servicios propios de un hotel de clasificación de dos y tres estrellas.

\section{CONCLUSIÓN}

Como es evidente, el sector hotelero es el más activo en el plan general del turismo, sin embargo no es valorizado de esa forma por algunos prestadores del servicio por lo que se debe hacer conciencia en la necesidad de un comportamiento respetuoso por el sector y los beneficios que ello generaría.

Es evidente la falta de organización y unificación de estrategias para atraer más turismo, brindarle nuevos y mejores servicios. Cada administración realiza acciones diferentes enfocadas principalmente al mantenimiento básico de la infraestructura del establecimiento y dejan a un lado acciones para implementar nuevos servicios y se mejore la calidad de los servicios ya que brindan, actualizar el método de publicidad (mercadotecnia) que manejan para llegar a nuevos huéspedes de una forma más eficiente y rápida. Implementar nuevos servicios aún es rechazado por considerarse un gasto innecesario y no una inversión.

Tras el estudio realizado, es indudable que los hoteles de dos y tres estrellas, aunque carezcan de los servicios básicos tendrán cupo lleno debido a los precios que manejan y sobre todo en la zona en donde se localizan, sin embargo lo que no es claro es por cuánto tiempo dichas características cubrirán las necesidades de los huéspedes. La competencia crece día a día, pero no se puede ver de una forma negativa, sino de forma constructiva para esforzarse más dentro de su establecimiento con nuevas tendencias, servicios que hagan que el huésped prefiera el establecimiento o recomiende por ser diferentes $\mathrm{y}$ originales.

\section{RECOMENDACIONES}

Contactar autoridades competentes para solicitar capacitación actualizada en cuanto a nuevas tecnologías para mejorar los servicios hoteleros.

Invertir en nuevos servicios como publicidad y tecnología (softwares, sistemas electrónicos del servicio, página de internet, etcétera) sobre las nuevas tendencias hoteleras y lo apliquen a sus negocios para aumentar la demanda y brindar servicios de calidad. 
Elorza et al., 2020

\section{LITERATURA CITADA}

Blesa Crucefix, P. L. 2017. Estrategias para hoteles independientes en Barcelona. pblesa@uoc.edu 10 de 2017. Consultado en la página:

http://miguelangelherrera.com/catedras/ad ministracion-mercadotecnia/dmpk.pdf

Caballero, S. 2018. La Importancia de la mercadotecnia en el turismo. Consultado en la página: http://www.milenio.com/opinion/variosautores/universidad-tecnologica-del-valledel-mezquital/la-importancia-de-la-

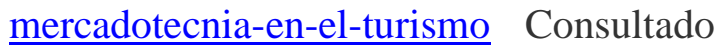
110918.

Fisher, L.; Espejo, J. Mercadotecnia, 2011. (P. 264). Cuarta Edición. Editorial Mc Graw Hill. Isbn 978-607-15-0539-2.
Herrera, Miguel Ángel. 20180. El Libro de la Mercadotecnia. Consultado en la página: https://www.academia.edu/18897949/Lib ro_Mercadotecnia_Laura_Fischer_y_Jorg e_EspejoFisher y espejo consultado el 14 de septiembre

Miranda, Vallejo. R. 2018. "Posicionamiento de las empresas turísticas en el puerto de Veracruz, caso: hotel playa paraíso "tesina. p 45-47. Consultado en la página: http://www.redalyc.org/jatsRepo/1934/19 3449985013/index.html

Miranda Z., y Estrada I. 2016. Redes sociales: herramienta de marketing internacional en el sector hotelero El Periplo Sustentable, núm. 31, 2016 Universidad Autónoma del Estado de México. Consultado en la página:

https://dialnet.unirioja.es/servlet/articulo? $\underline{\text { codigo }=3686039}$

Copyright (c) 2019 Elorza Martinez Oralia, Flores Barrios Leonardo, Gómez Priego Ilean A

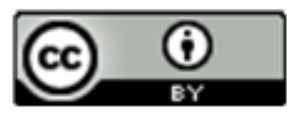

Este texto está protegido por una licencia licencia CreativeCommons 4.0.

Usted es libre para Compartir — copiar y redistribuir el $\mathrm{m}$ aterial en cualquier medio o formato- y Adaptar el documento — remezclar, transformar y crear a partir del material- para cualquier propósito, incluso para fines com erciales, siempre que cumpla la condición de:

Atribución: Usted debe dar crédito a la obra original de manera adecuada, proporcionar un enlace a la licencia, e in dicar si se han realizado cambios. Puede hacerlo en cualquier forma razonable, pero no de forma tal que sugiera que tiene el apoyo del licenciante o lo recibe por el uso que hace de la obra.

Resumendelicencia - Textocompletodelalicencia 